\title{
EL CORAJE DEL SUJETO
}

\section{(THE COURAGE OF THE SUBJETC)}

\section{Alejandro Granados-García}

(Pontificia Universidad Javeriana, Colombia) alejogranadosgarcia@gmail.com

Manuel Guerrero-Martelo

(Universidad Cooperativa de Colombia, Colombia) manuelfguerrero@gmail.com

Recibido: $14 / 10 / 2017$

Aprobado: 9/1/2018

"Una muchedumbre homogénea no reclama libertad. La sociedad jerarquizada no es meramente la única donde el hombre puede ser libre, sino también la única donde le urge serlo"

Nicolás Gómez Dávila

\begin{abstract}
RESUMEN
En este artículo se propone un abordaje de la cuestión del sujeto, a partir de una lectura del proyecto de pensamiento que Michel Foucault formuló y desarrolló hacia el final de su vida. Con este fin, se caracterizan, en primer lugar, las consideraciones y lineamientos centrales para el proyecto de una genealogía de la ética, materializado en una historia de la sexualidad. En orden de desarrollar esta línea de reflexión, se exponen, en un nivel general, los principales hallazgos de Foucault en el desarrollo de su historia de la sexualidad, jalonados por el feliz encuentro con las estéticas de la existencia. Posteriormente, se aborda la relación entre la genealogía de la ética y el ejercicio de la crítica, a partir de la comprensión de Foucault sobre la pregunta de Kant por la Ilustración. Para concluir, se reflexiona sobre la fascinación que produjo en Foucault su retorno a la antigüedad grecolatina y su apuesta por el coraje del sujeto.
\end{abstract}

Palabras claves: Foucault, sujeto, ética, poder, estética de la existencia.

\section{ABSTRACT}

This article proposes an approach to the question of the subject, based on a reading of the thought project that Michel Foucault formulated and developed towards the end of his life. To this end, the central considerations and guidelines for the project of a genealogy of ethics, materialized in a history of sexuality, are characterized in the first place. In order to develop this line of reflection, Foucault's main findings in the development of his history of sexuality are exposed, at a general level, marked by the happy encounter with the aesthetics of existence. Subsequently, the relationship between the genealogy of ethics and the exercise of criticism is addressed, based on Foucault's understanding of Kant's question for the Enlightenment. To conclude, we reflect on the fascination that Foucault produced in his return to Greco-Roman antiquity and his commitment to the courage of the subject. 
Keywords: Foucault, subject, ethics, power, aesthetics of existence.

\section{El proyecto de una genealogía de la ética}

En El sujeto y el poder (2015a), Michel Foucault reconoce que la meta de sus trabajos durante los últimos veinte años de su vida no se dirigió hacia una analítica del poder, sino a la producción de "una historia de los diferentes modos por los cuales, en nuestra cultura, los seres humanos son constituidos como sujetos” (p. 318). Con este fin, investigó tres modos de objetivación que transforman a los seres humanos en sujetos, a saber: la objetivación del sujeto mediante los saberes, mediante las prácticas divisorias y el modo en que un individuo se constituye a sí mismo en sujeto.

La cuestión del sujeto, como núcleo de las investigaciones de Foucault, demandaba un ajuste en la analítica del poder, aún más si se reconoce que la subjetividad no puede pensarse constituida enteramente solo por instancias exteriores, a la manera de un pliegue de la historia, los saberes y el poder. Se trata de una reivindicación del sujeto y sus posibilidades de des-subjetivarse, de asumir autónomamente su constitución y de ejercer prácticas de libertad y emancipación. Este ajuste conduce a que el poder se comprenda entonces en términos de gobierno.

Esta comprensión del ejercicio del poder y sus relaciones implica modos de acción que operan "sobre uno o varios sujetos actuantes y sobre lo que hacen o son capaces de hacer” (Foucault, 2015a, p. 334). Frente al comportamiento de los sujetos, el ejercicio del poder "incita, induce, disuade, facilita o hace más difícil, amplía o limita, vuelve más o menos probable” (ibídem). Gobernar, en este sentido, significa conducir y modificar conductas, es decir, estructurar un campo posible de acción.

Resulta fundamental, para la comprensión de la cuestión del sujeto, tener en cuenta que en la caracterización del poder como gobierno, se contempla la libertad como un elemento constitutivo. El poder, dice Foucault (2015a), no se ejerce más que sobre sujetos libres, entendiendo por ello: "sujetos individuales o colectivos que tienen ante sí un campo de posibilidades, donde pueden tener lugar varias conductas, varias reacciones y diversos modos de comportamiento” (p. 335). Allí donde las determinaciones están saturadas no hay relaciones de poder. Así, la libertad "aparece también como lo que no podrá más que oponerse a un ejercicio del poder que tiende a fin de cuentas a determinarla enteramente" (ibidem).

Esta reivindicación de la libertad, sumada al objetivo de Foucault de dar cuenta del modo en que un ser humano se convierte a sí mismo en sujeto, permite reconocer que la analítica del poder no solamente puede aplicarse al gobierno de la conducta de otros, sino al gobierno de la propia conducta, al gobierno de sí. Ante este escenario, Foucault enfoca su trabajo investigativo en trazar una genealogía del modo como en la cultura occidental, las personas han gobernado su propia conducta. Esto se traduce en una historia de la ética, entendiendo la ética como la "elaboración de una forma de relación consigo mismo que permite al individuo constituirse en sujeto de una conducta moral” (Foucault, 2011, p. 269). En otras palabras, se trata de una historia de las reflexiones, las técnicas y las prácticas a través de las cuales ha sido posible construir, autónomamente, la propia subjetividad.

Siguiendo esta línea de reflexión, el proyecto de una historia de la ética llevó a Foucault a desplazar la mirada hasta la antigüedad clásica, enfocándose en el dominio de la sexualidad, para "ver cómo y a través de qué formas concretas de relación consigo mismo, el individuo se ha visto ante la exigencia de constituirse como sujeto moral de conducta sexual” (Foucault, 2014, p. 21). Su proyecto se estructuró alrededor de una pregunta central: “¿por qué se hace del comportamiento sexual una cuestión moral, y una cuestión moral importante?” (Foucault, 2015b, p. 345). Ahora, Foucault (2015b) aclara que su interés se dirigía, especialmente, hacia "las técnicas de sí que permiten asegurar el dominio sobre los placeres y los deseos" (ibidem); modelando las relaciones consigo mismo y los demás, en "lo que puede denominarse pragmática de sí” (Foucault, 2014, p. 21). 
En la estructuración de su proyecto de una historia de la sexualidad, Foucault definió un "modelo analítico" compuesto por cuatro aspectos de la relación consigo mismo y unas regiones de la experiencia, que dan cuenta de las formas en que se problematizó el comportamiento sexual como asunto moral en la antigüedad grecorromana. Los aspectos de la relación consigo mismo, que determinan cómo debe constituirse el individuo en cuanto sujeto moral de sus propias acciones, son:

1) La sustancia ética, entendida como la parte de uno mismo o el comportamiento que está en relación con una conducta moral. Para los griegos la sustancia ética de la conducta sexual consistía en actos ligados en su unidad al placer y al deseo, esto es, las aphrodisia (actos, gestos, contactos, etc.);

2) El modo de sujeción, en virtud del cual los individuos tienen que reconocer las obligaciones morales que se les imponen (ley divina, natural, racional, principio estético de la existencia, etc.);

3) Las técnicas y prácticas de sí, es decir, los medios por los cuales podemos transformarnos a fin de llegar a ser sujetos morales. Se trata de las herramientas para ejercer un trabajo sobre la sustancia ética;

4) Una teleología moral, que establece la clase de sujeto en que queremos convertirnos cuando tenemos un comportamiento moral (libre, puro, bueno, dueño de sí mismo, etc.).

Estos aspectos de la relación con uno mismo y los demás son analizados en el marco de unas regiones determinadas de la experiencia, que generan inquietud y que son objeto de reflexión y problematización $^{1}$. Dichas regiones de la experiencia, que implican un haz de relaciones concretas, son:

1) la Dietética (relaciones con el cuerpo, el problema de la salud, la vida y la muerte);

2) la Económica (relaciones con el otro sexo, con el tema del matrimonio, el lazo conyugal y el gobierno del oikos);

3) la Erótica (relaciones con el propio sexo, con los muchachos, con el problema de ajuste entre papeles sociales y papeles sexuales) y;

4) la Verdad (relaciones con las condiciones “espirituales” que permiten acceder a la sabiduría).

El volumen sobre El uso de los placeres está consagrado a la forma en que la actividad sexual fue problematizada en esas regiones de la experiencia, por parte de filósofos y médicos en la cultura griega clásica del siglo IV a.C. Por su parte, el volumen sobre La inquietud de sí está consagrado a esta problematización en los textos griegos y latinos de los dos primeros siglos del imperio. El campo documental que analiza Foucault está constituido por textos de carácter "prescriptivo", "práctico”, que pretenden proponer reglas de conducta, opiniones y consejos para dirigir el comportamiento propio y de los demás. Al respecto, afirma Foucault (2011) que estos textos tienen como función "ser operadores que permitan a los individuos interrogarse sobre su propia conducta, velar por ella, formarla y darse una forma a sí mismos como sujetos éticos” (pp. 18-19); revelan, en suma, una función "eto-poética”, siguiendo a Plutarco.

\footnotetext{
${ }^{1} \mathrm{Si}$ la inquietud, la problematización y la reflexión en torno a la conducta sexual alcanzó la importancia que refleja la investigación de Foucault, esto se debe a que en la cultura grecolatina se reconocía la sexualidad como un campo agonístico de fuerzas de la naturaleza, virtualmente excesivas y difíciles de domeñar. Razón por la cual, la cuestión moral implicaba saber cómo hacer frente a esta fuerza de la naturaleza, para dominarla, asegurar su conveniente economía (cantidad y momento oportuno) y evitar que perturbe y amenace la relación del individuo consigo mismo y su constitución como sujeto moral.
} 


\section{El feliz encuentro con las estéticas de la existencia}

En el recorrido por la historia de la sexualidad, a Foucault le impresionó la relativa estabilidad de los códigos de restricciones y prohibiciones a través del tiempo. Sin embargo, encontró movimientos y transformaciones en la manera como se integraban esas restricciones en las relaciones consigo mismos. Este resulta un hallazgo fundamental. Nuestro filósofo encuentra que en la antigüedad grecorromana, en torno a las cuatro regiones inquietantes de la experiencia, se "desarrollaron artes de vivir, de comportarse y 'usar los placeres’ según principios exigentes y austeros” (p. 267).

Foucault da cuenta que la reflexión moral de la antigüedad, a propósito de los placeres, no se orientó hacia una codificación de los actos, ni buscaba la justificación e interiorización de interdicciones generales impuestas a todos, antes bien, se trató de una estilización de la actitud, del ejercicio del poder y la práctica de la libertad, en el marco de una estética de la existencia. Por esto último hay que entender:

\footnotetext{
Una manera de vivir cuyo valor moral no obedece ni a su conformidad con un código de comportamiento ni a un trabajo de purificación, sino a ciertas formas o más bien ciertos principios formales generales en el uso de los placeres, en la distribución que se hace de ellos, en los límites que se observan, en la jerarquía que se respeta (...) una vida así recibe el resplandor de una belleza manifiesta a los ojos de quienes pueden contemplarla o conservar su recuerdo (Foucault, 2011, p. 100).
}

Una estética de la existencia se traduce en prácticas voluntarias por las que los hombres no sólo se fijan reglas de conducta, sino que "buscan formarse a sí mismos, modificarse en su ser singular y hacer de su vida una obra que presenta ciertos valores estéticos y responde a ciertos criterios de estilo" (Foucault, 2011, pp. 16-17). El criterio estético que guiaba la configuración ética del sujeto, reclamaba una actitud (enkrateia) que designaba la forma de relación con uno mismo junto con las técnicas y prácticas que soportaban esa relación. Se trataba de una forma activa de dominio de uno mismo, que permitía luchar para asegurar la templanza (sophrosyne), el gobierno de sí, ante los deseos y placeres. El objetivo de este gobierno de sí era "ser libre y poder seguir siéndolo” (Foucault, 2011, p. 87). La libertad significa, para cada uno, una determinada forma de relación de gobierno, de soberanía, del individuo sobre sí mismo.

En el núcleo de una estética de la existencia en la antigüedad grecorromana se encontraba la epimeleia heautou, un ejercicio práctico del cuidado de sí que representaba una de las reglas o prescripciones más importantes para la conducta social e individual. En este ejercicio se articulaba el conocimiento, la atención, la ocupación, el cultivo y las técnicas de sí. Ese cultivo de sí hacía de la estética de la existencia una techné tou biou, tomando la forma de:

\footnotetext{
Una actitud, de un modo de comportarse que impregna las maneras de vivir y se desarrolló en procedimientos, en prácticas y recetas que se meditan, perfeccionan y enseñan: constituyó una práctica social que generó relaciones interindividuales, intercambios y comunicaciones $\mathrm{y}$, a veces, incluso instituciones; dio lugar, finalmente, a cierto modo de conocimiento y a la elaboración de un saber (Foucault, 2010a, p. 51).
}

Por lo anterior y por la centralidad de la figura de Alcibíades para Foucault, se entiende que el cuidado de sí no resultaba un ejercicio individualista, reclamaba, a su vez, el cuidado, el cultivo y el gobierno de los otros en la vida cotidiana. En otras palabras, "el cuidado de sí (...) aparece como una intensificación de las relaciones sociales (...) comprende la posibilidad de un juego de intercambios con el otro y un sistema de obligaciones recíprocas” (Foucault, 2010a, p. 62). Esto resulta importante, pensando en responder a posibles críticas de individualismo esteticista o incluso de dandismo, que se puedan formular a la idea de una estética de la existencia como eje de la configuración de un sujeto ético. 


\section{La genealogía de la ética y el ejercicio de la crítica}

Para establecer el significado y el valor que Foucault encontró en su retorno a la antigüedad grecorromana, es necesario abordar brevemente la lectura que hace Foucault del texto de Kant (1784) sobre la Ilustración (Wass heisst Aufklärung?). Según se lee en El gobierno de sí y de los otros, Foucault (2014) encuentra en este texto la pregunta por la actualidad que lleva al análisis de los que somos, de nuestro tiempo y del campo actual de nuestras experiencias posibles. Se trata de una ontología del presente. En este escenario se inscribe la cuestión del sujeto perseguida por Foucault ${ }^{2}$.

Para Kant la Ilustración significa la salida del hombre de la minoría de edad de la que él mismo es responsable. Minoría de edad que se traduce en la incapacidad de valerse del entendimiento sin la dirección de otros. Su causa la encuentra Kant en la pereza, la falta de decisión y de coraje para valerse por sí mismo, siendo por esto el lema de la Ilustración jsapere aude! Este coraje que se demanda hace pensar en la enkrateia que se prescribía en la antigüedad grecorromana. Se trata de la actitud necesaria para impulsar y sostener el gobierno de sí, que estaría centrado para Kant en el uso (público y privado) que damos a nuestra razón.

De esta forma, la genealogía de la ética, se articula con la tradición crítica inaugurada por Kant, para dar cuenta de los límites de aquello que somos en nuestra actualidad. Se trata de una actitud que abre posibilidades para problematizar los modos en que históricamente hemos sido gobernados y nos hemos gobernado a nosotros mismos, esto, si se quiere, con el fin de franquear límites, de generar formas de des-subjetivación, prácticas de liberación, que permitan proyectar distintas maneras de constituirnos como sujetos.

\section{La fascinación de Foucault por el coraje del sujeto}

Si la apuesta de Foucault es por una ontología del presente y reconoce su desconfianza hacia la idea de un sujeto universal, ¿qué sentido tiene su retorno a la antigüedad y qué fue lo que le fascinó a su regreso?

Así expresa Foucault (2005) la fascinación que le produjo el escenario que encontró en su retorno a la antigüedad grecorromana: "La idea del bios como material de una obra de arte es algo que me fascina. También la idea de que la moral puede ser una estructura muy fuerte de existencia sin estar ligada a un sistema autoritario ni jurídico en sí, ni a una estructura de disciplina” (p. 489). Frente a esto último y en el marco de la crítica de la actualidad, nuestro filósofo reconoce que si se interesó en la antigüedad, es debido a que "por toda una serie de razones, la idea de una moral como obediencia a un código de reglas está, ahora, en trance de desaparecer. Y a esta ausencia de moral responde, debe responder, una búsqueda que es la de una estética de la existencia” (Foucault, 2015c, p. 373) A esto añade: "somos mayoría los que no creemos que una moral pueda fundarse en la religión y no queremos que un sistema legal intervenga en nuestra vida moral, personal e íntima” (2015b, p. 346).

Para concluir, en su postura crítica ante nuestra actualidad, Foucault (2015b) afirma:

Lo que me sorprende es que en nuestra sociedad el arte ya solo tenga relación con los objetos y no con los individuos o la vida; y también que el arte sea un dominio especializado, el dominio de los expertos que son los artistas. Pero ¿no podría la vida de cualquier individuo ser una obra de arte? ¿Por qué un cuadro o una casa son objetos de arte, pero no nuestra vida? (p. 353)

\footnotetext{
${ }^{2}$ Resulta fundamental tener en cuenta la siguiente consideración de Foucault (2015c), que ayuda a trazar límites claves para pensar la cuestión del sujeto en su obra: "Pienso que no hay un sujeto soberano, fundador, una forma universal de sujeto que se podría encontrar por todas partes. Soy muy escéptico y muy hostil hacia esa concepción del sujeto. Pienso, por el contrario, que el sujeto se constituye a través de prácticas de sujeción, o, de una manera más autónoma, a través de prácticas de liberación, de libertad, como en la Antigüedad, a partir, claro está, de un cierto número de reglas, estilos, convenciones, que se encuentran en el medio cultural” (p. 375).
} 
Su fascinación por el feliz encuentro con las estéticas de la existencia en la antigüedad, refleja entonces la apuesta por ampliar ética y estéticamente el campo actual de nuestras experiencias posibles. Sin embargo, es necesario aclarar que Foucault no regresa a la antigüedad para encontrar una solución de recambio o el contenido de un modelo ético concreto para el presente. Antes bien, rastrea un principio ético-estético que permita aprender a gobernarnos, con el coraje necesario (la enkrateia) para asumir una posición autónoma ante las exigencias de los códigos morales y desplegar prácticas de libertad, de cultivo y cuidado de sí y de los otros.

Foucault hace suyo este principio ético-estético y lo expresa en la respuesta que da a la pregunta de Dreyfus y Rabinow sobre ¿qué vendrá a continuación?, a lo que responde: “¡Ah, ante todo voy a ocuparme de mí mismo!” (Foucault, 2015b, p. 346). Se trata de una doble referencia, autobiográfica y a su proyecto genealógico, en la que la transformación de la propia vida aparece como una condición para el ejercicio del pensamiento. Precisamente, en la comprensión de los cínicos que expone en su último curso en el Collège de France (El coraje de la verdad, 2010b), se encuentra que la manifestación de la verdad ya no se inscribe simplemente a través de una toma arriesgada de la palabra, sino en el espesor mismo de la existencia. La propia vida se erige como escenario de verificación, de comprobación de la verdad del discurso (relación entre logos y bios).

En consecuencia, el ejercicio de una crítica de la actualidad resulta insuficiente si se dirige exclusivamente a las estructuras y dinámicas sociales de gobierno y explotación. Es indispensable dirigir la crítica hacia nosotros mismos, hacia nuestras formas de subjetivación. Encontramos entonces en el abordaje de Foucault sobre la cuestión del sujeto, en sus últimos años de vida, una reivindicación del mismo, procurando rescatarlo de su reducción a la condición de pliegue de la historia, del saber y del poder. Esto representa la apuesta por ampliar el campo de posibilidades de nuestra experiencia y del gobierno de sí, estableciendo una relación ético-estética con uno mismo y los demás. Es la apuesta por prácticas de autonomía, de des-subjetivación, de libertad; sin desconocer el marco normativo actual en el que somos gobernados, pero problematizándolo. Se trata de una invitación a escuchar el eco del canto grecolatino por el cuidado y el cultivo de sí. Una invitación a tener el coraje para erigirnos como estetas-éticos de nuestra existencia y convertir nuestra vida en una obra de arte.

\section{Referencias}

FOUCAULT, M. (2005). La hermenéutica del sujeto. Madrid: Ediciones Akal.

--- (2010a). Historia de la sexualidad. La inquietud de sí (V.3). México: Siglo XXI.

--- (2010b). El coraje de la verdad. El gobierno de sí y de los otros II. Buenos Aíres: Fondo de Cultura Económica.

--- (2011). Historia de la sexualidad. El uso de los placeres (V.2). México: Siglo XXI.

--- (2014). El gobierno de sí y de los otros. Buenos Aíres: Fondo de Cultura Económica.

--- (2015a). El sujeto y el poder, 1983. En La ética del pensamiento: para una crítica de lo que somos. Madrid: Biblioteca Nueva.

--- (2015b). Acerca de la genealogía de la ética. Un panorama del trabajo en curso, 1983 (Entrevista con H. Dreyfus y P. Rabinow). En La ética del pensamiento: para una crítica de lo que somos. Madrid: Biblioteca Nueva.

--- (2015c). Una estética de la existencia, 1984 (Entrevista con A. Fontana). En La ética del pensamiento: para una crítica de lo que somos. Madrid: Biblioteca Nueva.

GÓMEZ DÁVILA, N. (2005). Escolios a un texto explicito I. Bogotá: Villegas Editores. 